\title{
Direct and disequilibrium effects on precipitation in transient climates
}

\author{
D.J McInerney and E.J Moyer
}

\section{Supplementary Material}

\section{A. CCSM3 model configuration and experiments}

Climate simulations were made with the low resolution version of the Community Climate System Model (CCSM3) with T31 atmospheric resolution [1,2]. Because the goal of the experiments was to understand transient climate responses, forcing changes were applied by varying a single parameter (atmospheric $\mathrm{CO}_{2}$ concentration, which is assumed globally uniform). Other greenhouse gases, sulphate aerosols, and all other model parameters are fixed at their pre-industrial values. To enhance our ability to detect signal from noise, we performed five simulations (realizations) of most experiments using different initial conditions. Initial conditions are taken from the NCAR b30.048 pre-industrial control run [2]; in particular, we used restart files from the years 410, 420, 430, 440 and 450 of this run. Experiments beginning from pre-industrial climate were initiated directly from the NCAR restart files. For experiments that begin in the year 2010, we spun up the climate by running each realization from 1870-2010 with historical $\mathrm{CO}_{2}$ concentrations before branching off into the different experiments in the year 2010.

The four CCSM3 experiments conducted in this study are

(a) Instantaneous $\mathrm{CO}_{2}$ increase: Atmospheric $\mathrm{CO}_{2}$ concentration instantly increases from the pre-industrial concentration of 289 ppm to 700 ppm (five realizations, 330 years).

(b) Instantaneous solar increase: Solar forcing instantly increases by $2.55 \%$, with $\mathrm{CO}_{2}$ concentrations remaining at $289 \mathrm{ppm}$. This change in solar forcing was chosen to replicate the global mean temperature change for the above $\mathrm{CO}_{2}$ scenario over the initial period (five realizations, 330 years).

(c) Equilibration after $\mathrm{CO}_{2}$ rise: $\mathrm{CO}_{2}$ concentrations rise gradually from 289 ppm to 700 ppm over the period 1870-2100 (230 years), then are stabilized and the run continues for $\sim 5000$ years. (Only one realization continues to equilibrium). 
(a)

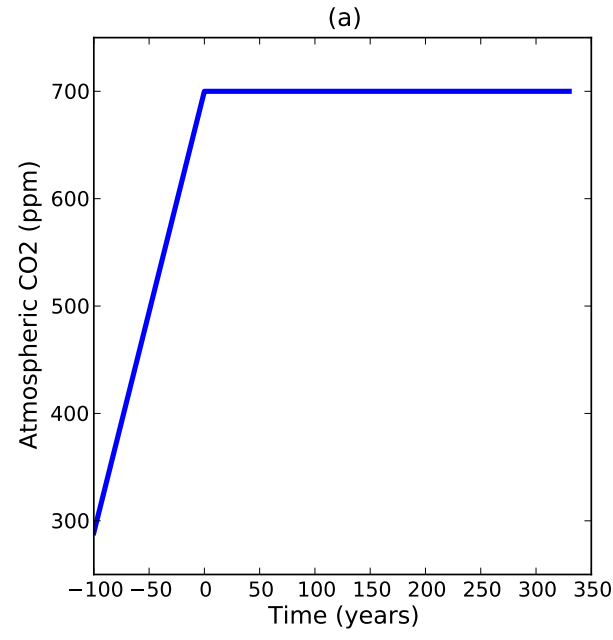

(c)

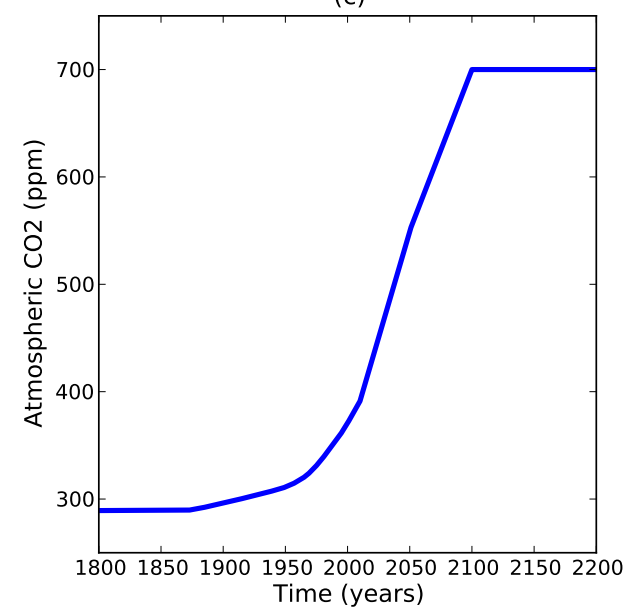

(b)

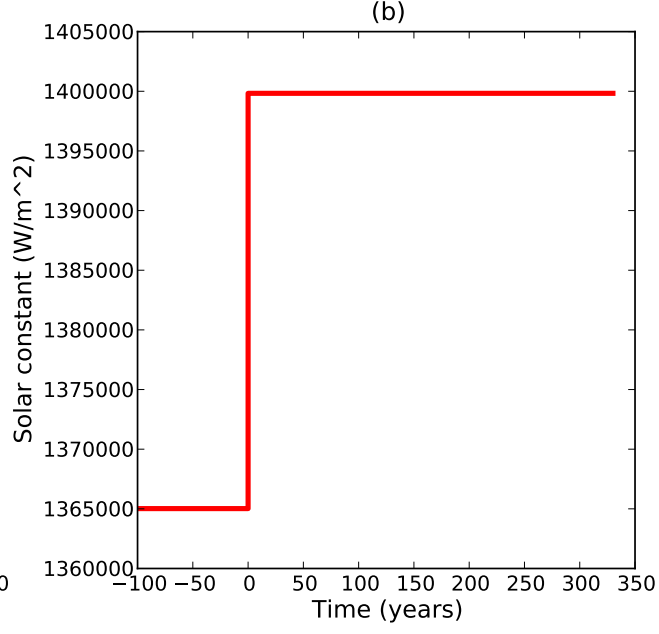

(d)

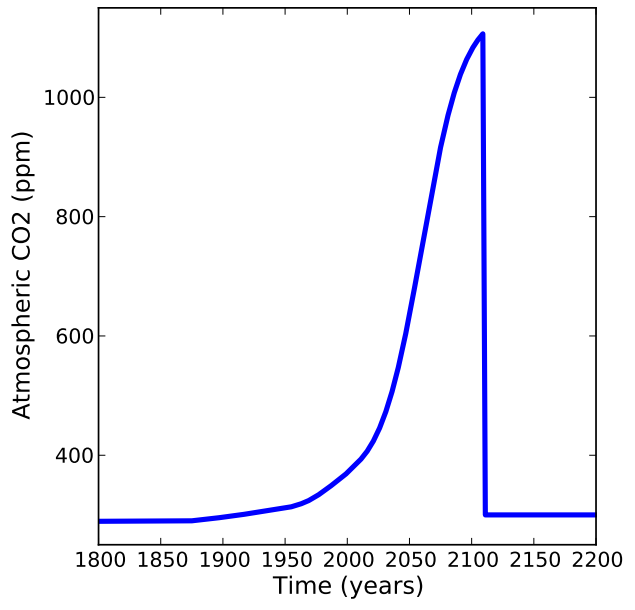

Supplementary Figure 1: Changes in forcing agent for the four CCSM3 experiments listed in Supp. Mat. Sec. A.

(d) Air capture scenario: $\mathrm{CO}_{2}$ concentrations follow historical concentrations between 1870 and 2010, increase rapidly from $391 \mathrm{ppm}$ in 2010 to $1100 \mathrm{ppm}$ in the year 2110 (following a logistic curve), then instantaneously drops to $300 \mathrm{ppm}$ in 2111 to simulate a sudden air capture (five realizations).

The changes in forcing agent for these experiments are displayed in Supp. Fig. 1. 


\section{B. CMIP3 "1pctto2x" experiment}

The multi-model mean temperature and precipitation anomalies over land and ocean shown in Figure 2 of the manuscript are derived from the 13 archived model runs of the CMIP3 "1pctto2x" experiment. Output of individual runs are are shown in Supp. Figs. 2. Larger internal variability is seen in land anomalies than ocean anomalies, and precipitation shows greater internal variability than does temperature. Inter-model differences are also greater for precipitation than temperature (Supp. Fig. 2).

We employ two statistical models to characterize the relationship between temperature and precipitation in our analysis of the CMIP3 stabilization experiments. To characterize the overall trend of precipitation with temperature over the entire pre- and post-stabilization period we fit GCM data to a simple linear model of the form

$$
P_{n}=\alpha+\beta T_{n}+\epsilon_{n} .
$$

To quantify the change in precipitation response after stabilization we fit a two-phase linear model

$$
\Delta P_{n}= \begin{cases}\alpha_{1}+\beta_{1} \Delta T_{n}+\epsilon_{n} & \text { if } n \leq s, \\ \alpha_{2}+\beta_{2} \Delta T_{n}+\epsilon_{n} & \text { if } n>s,\end{cases}
$$

where $t_{s}$ is the stabilization time; $\alpha_{1}$ is the precipitation anomaly corresponding to a zero temperature anomaly; $\beta_{1}$ and $\beta_{2}$ are the pre- and post-stabilization precipitation responses; and $\epsilon_{n}$ are residuals. For continuity, we set $\alpha_{1}+\beta_{1} T_{s}=\alpha_{2}+\beta_{2} T_{s}$; however, we do not constrain $\alpha_{0}$ to zero because the temperature and precipitation anomalies at the beginning of the simulations are not always zero (Supp. Fig. 2). Our two-phase model improves on that of [3] - who performed separate linear regressions on pre- and post-stabilization global temperature and precipitation data to determine precipitation responses for each period - by ensuring continuity in the precipitation response at the time of stabilization. This is particularly important when calculating the precipitation response over land, since land model output contains more internal variability.

The relationship between temperature and precipitation for individual models is shown for the ocean in Supp. Fig. 3 and for the land in Supp. Fig. 4. Over the ocean, most models show a clear increase in precipitation response $(\Delta P / \Delta T)$ after stabilization, with the only exception being GISS-EH. Over land, the two-phase linear model actually suggests that precipitation per warming decreases after stabilization in 12 out of 13 models (Supp. 

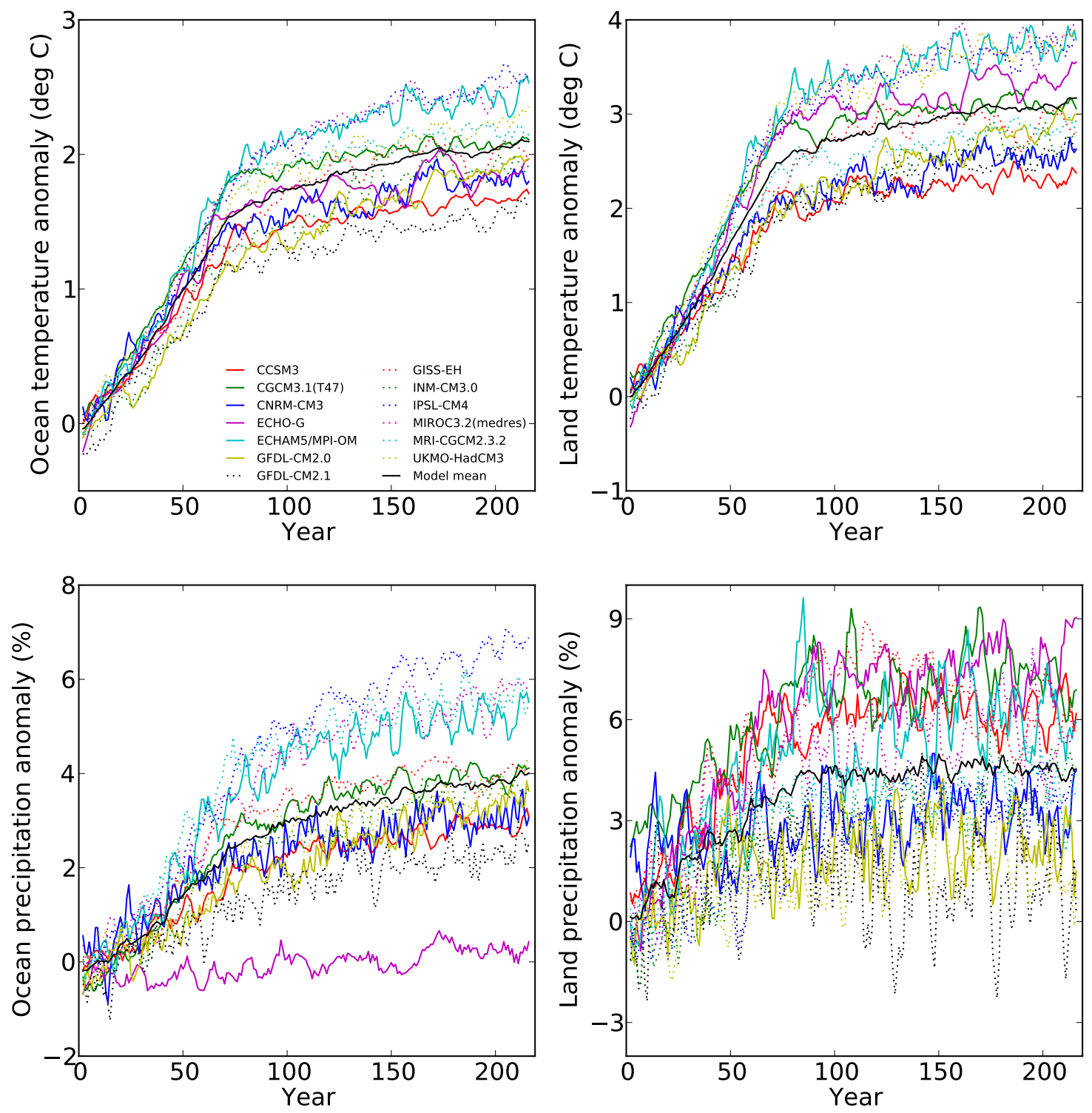

Supplementary Figure 2: Temperature and precipitation anomalies (after 5-year box-car smoothing) for the "1pctto2x" experiments from 13 models in the CMIP3 archive. Each experiment consists of only one realization.

Fig. 4), but we place less confidence in these model fits since the standard deviation of residuals between the two-phase model and the data are much greater over land than over the ocean (Supp. Table I). A single outlier among among the models for land precipitation is IPSL-CM4, which shows a distinct increase in precipitation per warming after stabilization. Derived values for pre- and post-stabilization responses (calculated using Supp. Equation 2) are given in Supp. Table I. 

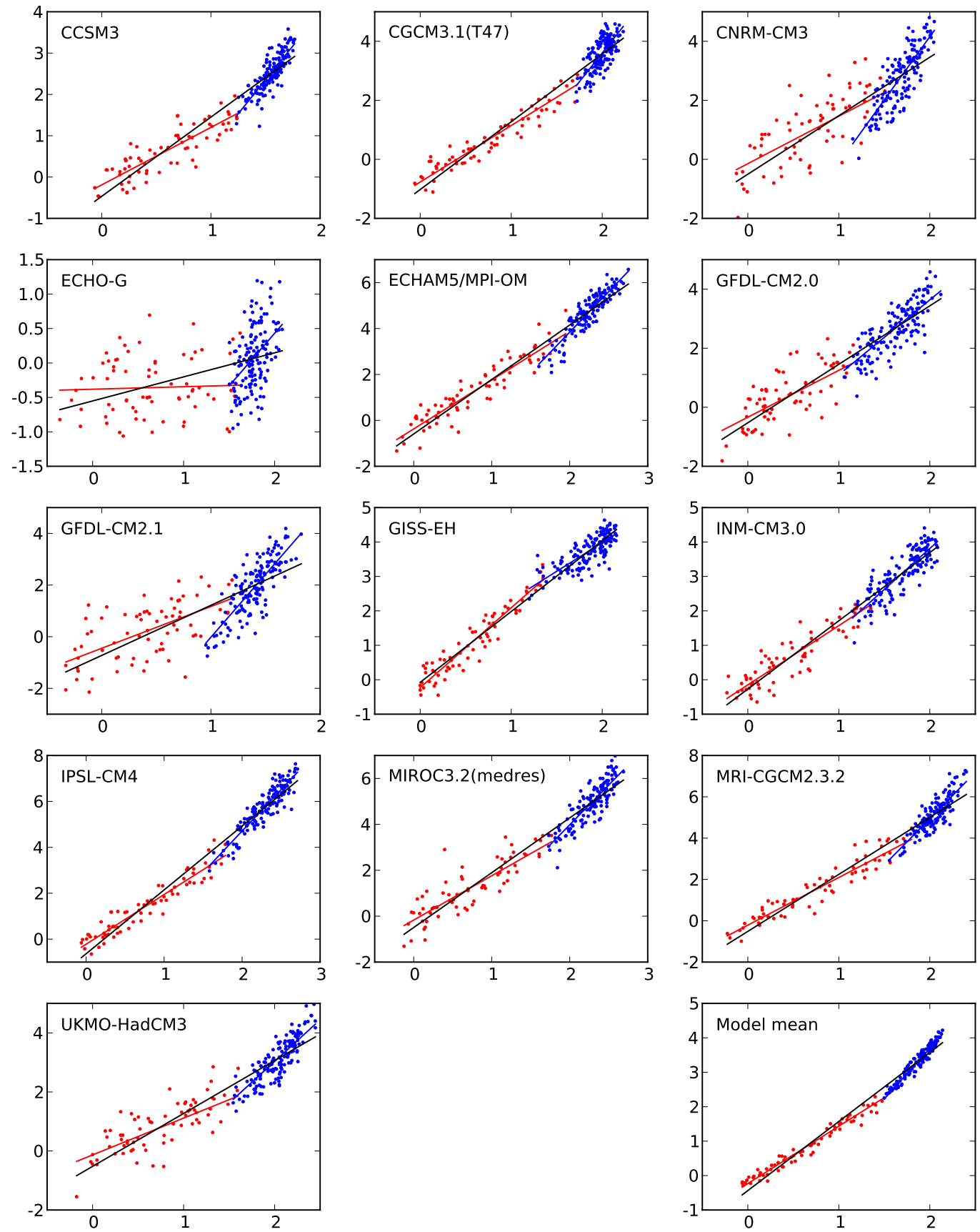

Supplementary Figure 3: Ocean temperature anomaly (K) versus ocean precipitation anomaly (\%) for the CMIP3 "1pctto2x" experiment". Individual panels correspond to the 13 models considered and the multi-model mean. Red and blue data and lines correspond to preand post-stabilization model output and fits from the two-phase linear model, while the black line is the single-phase linear model fit. 

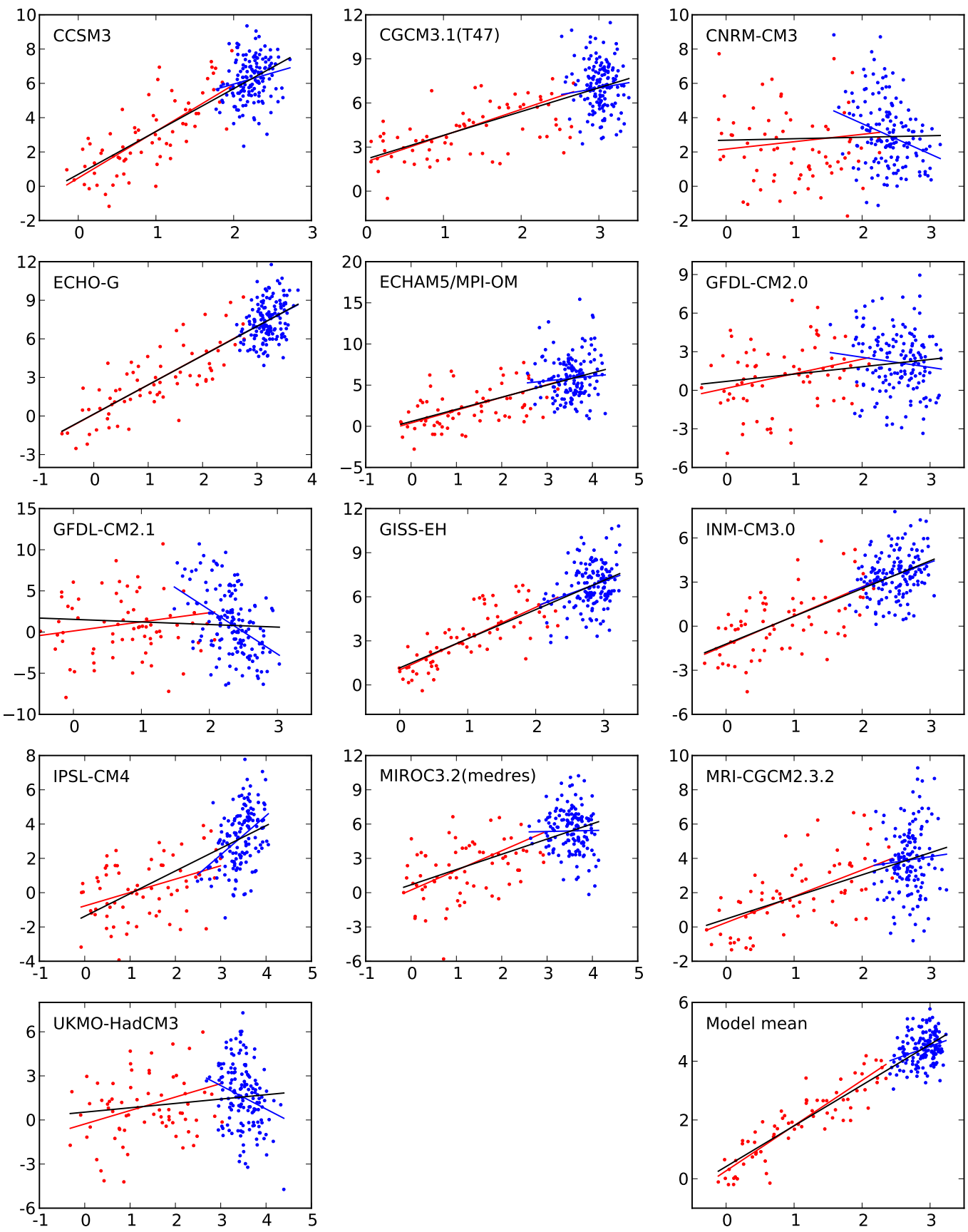

Supplementary Figure 4: Land temperature anomaly (K) versus land precipitation anomaly (\%) for the CMIP3 "1pctto2x" experiment. Individual panels correspond to the 13 models considered and the multi-model mean. Red and blue data and lines correspond to pre- and post-stabilization model output and fits from the two-phase linear model, while the black line is the single-phase linear model fit. 
Supplementary Table I: Pre- and post-stabilization precipitation responses in $\% /$ K, i.e. $\beta_{1}$ and $\beta_{2}$ in Supp. Equation (2), over land and ocean for the CMIP3 "1pctto2x" experiments. Standard errors are for the residuals between the two-phase linear model and GCM precipitation data.

\begin{tabular}{|l|c|c|c|c|c|c|}
\hline & \multicolumn{3}{|c|}{ Ocean } & \multicolumn{3}{c|}{ Land } \\
\hline Model & Pre-stab & Post-stab & Std err & Pre-stab & Post-stab & Std err \\
\hline CCSM3 & 1.39 & 3.28 & 0.27 & 2.72 & 1.31 & 1.28 \\
CGCM3.1(T47) & 1.92 & 3.89 & 0.37 & 1.78 & 0.92 & 1.60 \\
CNRM-CM3 & 1.61 & 4.28 & 0.64 & 0.43 & -1.77 & 1.94 \\
ECHO-G & 0.04 & 1.53 & 0.41 & 2.29 & 2.21 & 1.49 \\
ECHAM5/MPI-OM & 2.13 & 3.53 & 0.42 & 1.55 & 0.56 & 2.44 \\
GFDL-CM2.0 & 1.59 & 2.54 & 0.53 & 1.14 & -0.79 & 2.36 \\
GFDL-CM2.1 & 1.62 & 4.87 & 0.72 & 1.11 & -5.37 & 3.55 \\
GISS-EH & 2.32 & 1.66 & 0.29 & 2.13 & 1.70 & 1.35 \\
INM-CM3.0 & 1.72 & 2.38 & 0.35 & 1.98 & 1.69 & 1.55 \\
IPSL-CM4 & 2.16 & 3.61 & 0.38 & 0.78 & 2.28 & 1.54 \\
MIROC3.2(medres) & 1.93 & 3.47 & 0.50 & 1.72 & 0.09 & 2.03 \\
MRI-CGCM2.3.2 & 2.31 & 4.48 & 0.42 & 1.53 & 0.61 & 1.80 \\
UKMO-HadCM3 & 1.25 & 2.82 & 0.44 & 0.92 & -1.60 & 2.07 \\
\hline Model mean & 1.66 & 2.94 & 0.13 & 1.54 & 0.83 & 0.52 \\
\hline
\end{tabular}

Residuals between the single-phase linear model fits (black lines in Supp. Figs. 3 and 4) and the GCM precipitation are shown in Supp. Figs. 5 and 6. Over the ocean, residuals typically decrease until the time of $\mathrm{CO}_{2}$ stabilization, then increase post-stabilization. The characteristic "V" shape pattern is consistent amongst all models except GISS-EH, which (as mentioned previously) showed a decrease in precipitation response after stabilization. Over land, residuals of the individual models show no consistent pattern. 

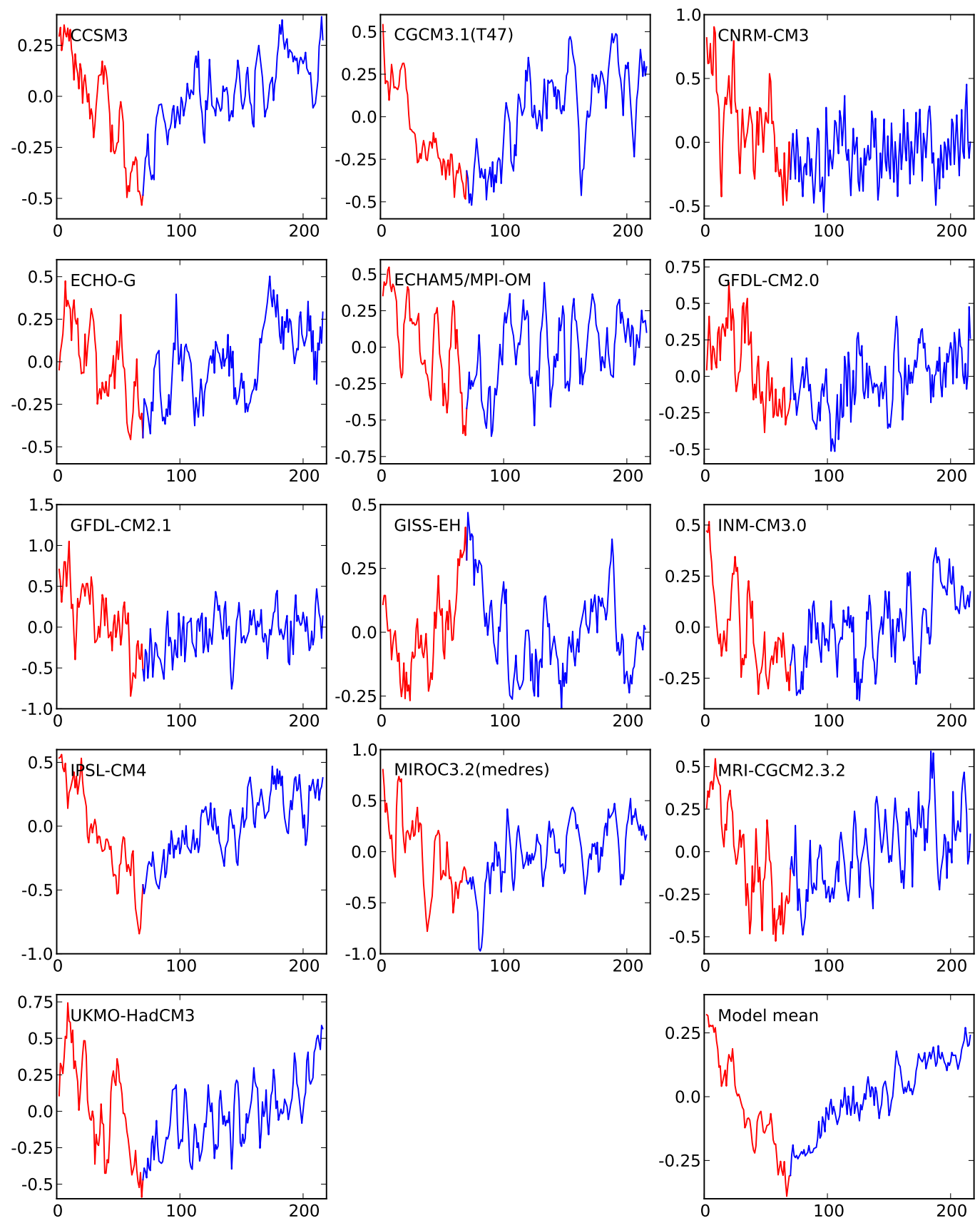

Supplementary Figure 5: Residuals between ocean precipitation (points in Supp. Fig. 3) and the single phase linear model (black lines in Supp. Fig. 3) for the CMIP3 "1pctto2x" experiment (after 5-year boxcar smoothing). 

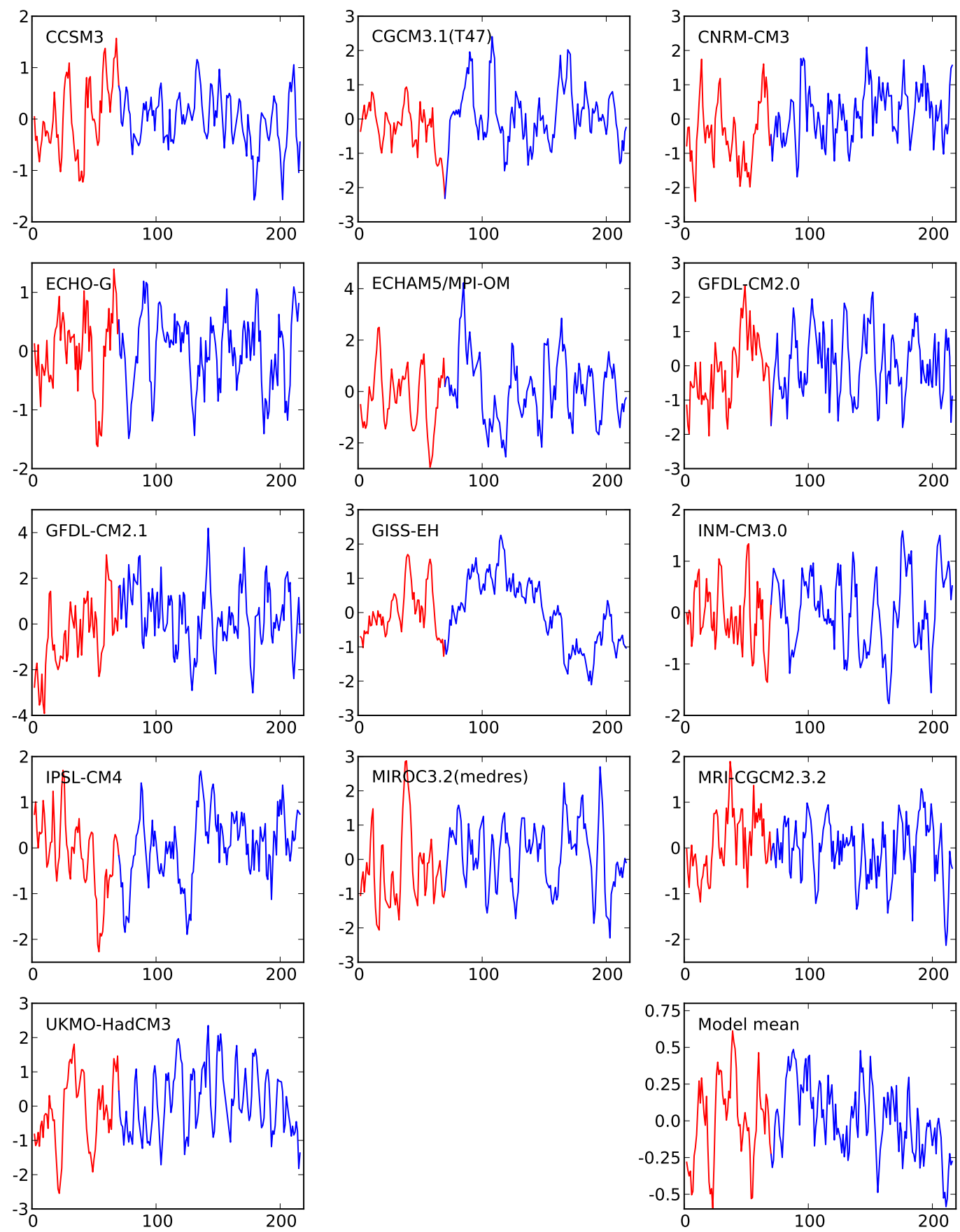

Supplementary Figure 6: Residuals between land precipitation (points in Supp. Fig. 4) and the single phase linear model (black lines in Supp. Fig. 4) for the CMIP3 "1pctto2x" experiment (after 5-year boxcar smoothing). 


\section{Regional coefficients}

In the manuscript we first introduce two representations of transient precipitation that are exactly analogous, the "disequilibrium" framework

$$
\Delta P=c_{1} \Delta T-c_{2}\left(\Delta T_{e q}-\Delta T\right)
$$

and the "fast-slow" framework

$$
\Delta P=\alpha \Delta T+\beta \Delta T_{e q} .
$$

In this expression, the transient term is a function of equilibrium temperature alone (though coefficients may vary between forcing agents).

In the literature, the transient term in the "fast-slow" framework is more commonly written in terms of the individual forcing agent, so that the formulation for $\mathrm{CO}_{2}$ forcing becomes

$$
\Delta P=\alpha \Delta T+\beta_{\mathrm{CO}_{2}} \log _{2}\left(\frac{\mathrm{CO}_{2}}{\mathrm{CO}_{2, \mathrm{PI}}}\right)
$$

where $\alpha$ is the coefficient of the temperature dependent term, and $\beta_{\mathrm{CO}_{2}}$ is the instantaneously change in precipitation for a doubling of atmospheric $\mathrm{CO}_{2}$ [4]. The analogous expression for transient precipitation evolution under changing solar forcing is

$$
\Delta P=\alpha \Delta T+\beta_{S} \Delta S .
$$

where $\beta_{S}$ is the instantaneous change in precipitation for each one percent change in solar forcing $(\Delta S)$. We show in the manuscript coefficients derived for equations (5) and (6), but also here for equation (4) to confirm that the choice of representation of the transient term in the "fast-slow" framework does not affect our overall conclusions. 


\section{Estimation of coefficients}

(a) Linear evolution

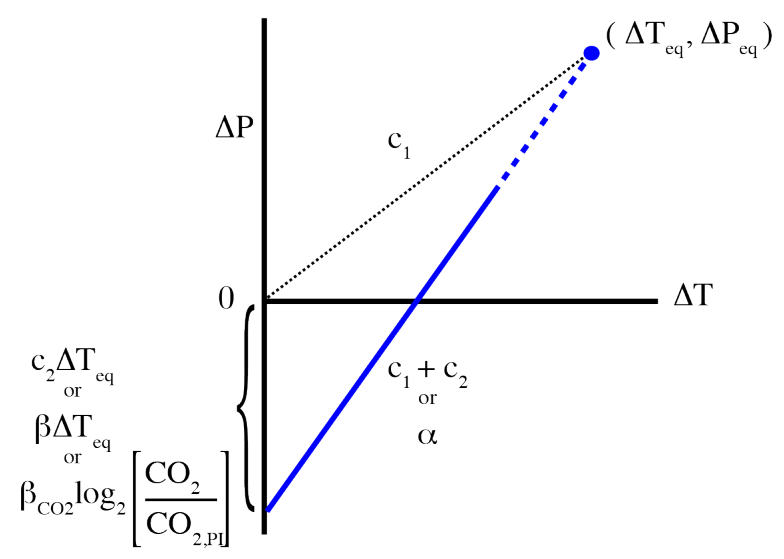

(b) Nonlinear evolution

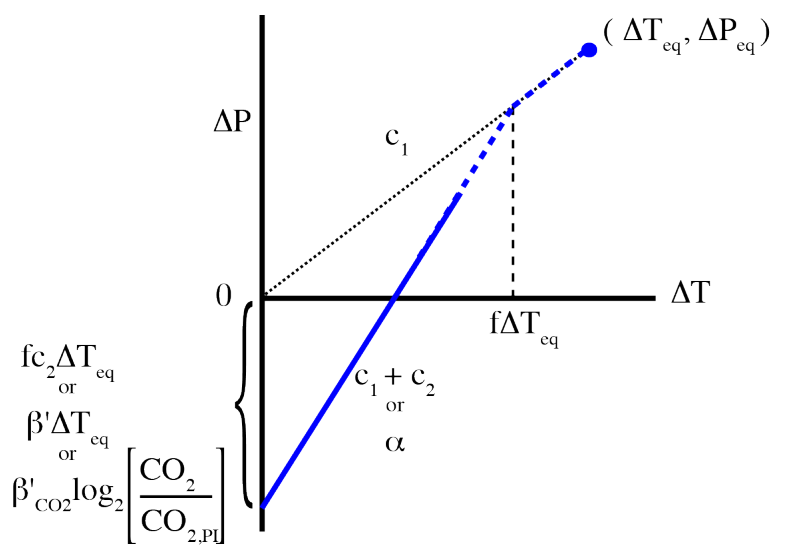

Supplementary Figure 7: Cartoons displaying the relationship between temperature and precipitation anomalies, and the means of deriving coefficients in Supp. Eqns. 3-5, for scenarios where forcing is abruptly changed. Panel (a) shows the case where precipitation remains linear with temperature change, while (b) shows a case where this relationship becomes nonlinear.

We compute grid-point values for the coefficients $\alpha$ and $\beta_{\mathrm{CO}_{2}}$ using data from GCM experiments where atmospheric $\mathrm{CO}_{2}$ concentration instantaneously rises. The $\alpha \mathrm{s}$ are the slopes of $\Delta P$ vs. $\Delta T$, derived from performing a linear regression on the initial evolution of $\Delta P$ at each grid-point. The $\beta_{\mathrm{CO}_{2}} \mathrm{~s}$ are the intercepts of this regression (Supp. Fig. $7 \mathrm{a}$ ). (Coefficients $\alpha$ and $\beta_{S}$ for solar-forced runs are derived in this way as well).

The coefficients $c_{1}, c_{2}$ and $\beta$ are derived using equilibrium temperature and precipitation anomalies $\left(\Delta T_{e q}\right.$ and $\left.\Delta P_{e q}\right)$. The $\beta$ s rely on knowledge of $\Delta T_{e q}$, but are taken from the intercepts (assumed $\beta \Delta T_{e q}$ ). The $c_{1}$ s are the equilibrium hydrological sensitivities at each model grid-point $\left(c_{1}=\Delta P_{e q} / \Delta T_{e q}\right)$. The $c_{2}$ s are taken from $c_{1}$ and from the initial slope, which is $c_{1}+c_{2}$ (Supp. Fig $\left.7 \mathrm{a}\right)$.

If the evolution of post-stabilization precipitation is linear with temperature, there is no distinction between deriving $c_{2}$ from slope or intercept (Supp. Fig 7 a). That linearity has been assumed in all previous studies (e.g. [3, 4]). However, our long equilibrated run has shown that long-term evolution of precipitation is slightly non-linear with temperature and follows behavior similar to that in Supp. Fig. 7(b). Initial precipitation evolution, if 
projected further, does not intersect the final equilibrium point $\left(\Delta T_{e q}, \Delta P_{e q}\right)$ and is better represented as

$$
\Delta P=c_{1} \Delta T-c_{2}\left(f \Delta T_{e q}-\Delta T\right)
$$

with $f \approx 0.7$. That is, coefficients $c_{2}$ would be globally reduced by $\approx 25 \%$ if taken from the intercepts rather than the slopes. The distinction does not affect the major findings of this paper, but should be noted as a source of potential confusion if researchers use differing approaches to deriving coefficients.
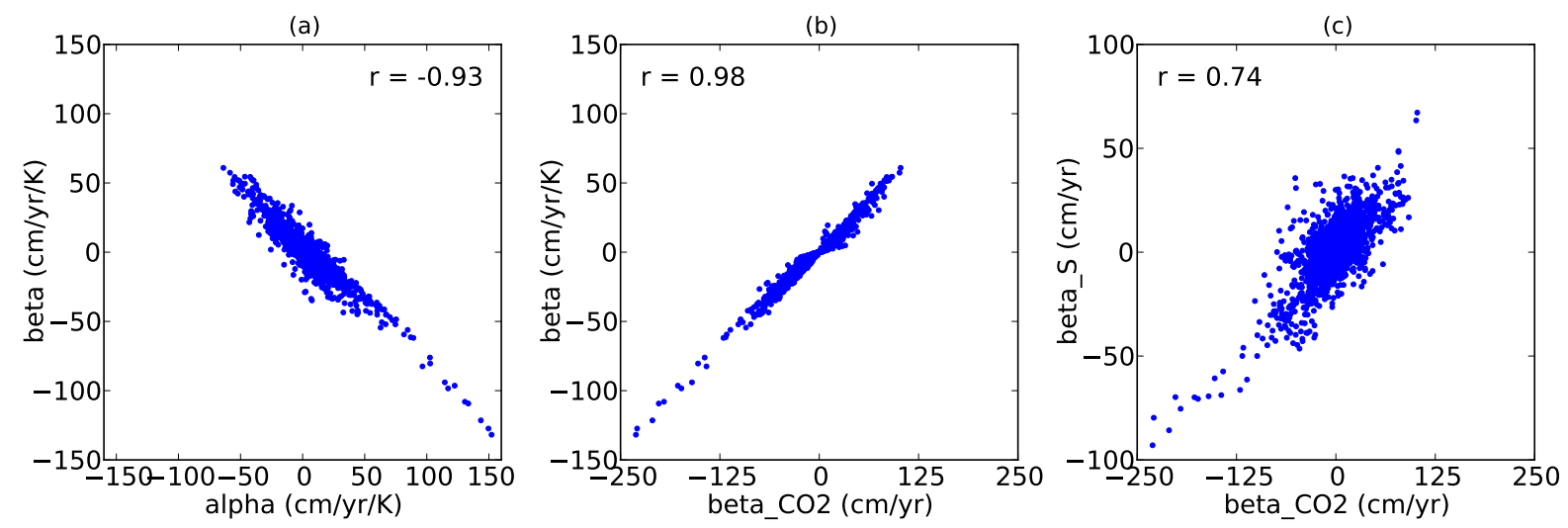

Supplementary Figure 8: Scatter plots showing the correlation between (a) $\alpha$ and $\beta$, (b) $\beta_{\mathrm{CO}_{2}}$ and $\beta$, and (c) $\beta_{\mathrm{CO}_{2}}$ and $\beta_{S}$. Each dot corresponds to a model grid point, while the $r$-value is Pearson's correlation coefficient.

In the manuscript we showed only the correlation between $\alpha$ and $\beta_{\mathrm{CO}_{2}}$ (Fig. $6 \mathrm{~b}$ ) and asserted that the substitution of $\beta_{\mathrm{CO}_{2}}$ and $\beta$ was not significant. We show here the equally strong correlation between $\alpha$ and $\beta$ (Supp. Fig. $8 \mathrm{a}, r=-0.93$ ), which results from the negligible differences between $\beta$ and $\beta_{\mathrm{CO}_{2}}$ compared to regional variation (Supp. Fig. 8 b, $r=$ -0.98). We noted in the manuscript that the pattern of transient precipitation response in solar-forced climates $\left(\beta_{S}\right)$ is similar to that in $\mathrm{CO}_{2}$-forced climates $\left(\beta_{\mathrm{CO}_{2}}\right)$. We demonstrate this in Supp. Fig. 8(c).

The period of regression has a small effect on the values derived for $\alpha, \beta_{\mathrm{CO}_{2}}$ and $c_{2}$ but again does not affect the findings of this study. For our coefficient fits for our CCSM3 runs with a fully coupled ocean, we use decadally averaged data between the years 10 and 80 . This period is shorter than the 150 years used by [3] to estimate the "slow" response using 
CMIP3 data from fully coupled models. (We did use the 150 year period in Section 3 of the manuscript, where we reproduce the [3] analysis for land and ocean separately). For coefficient evaluation, we chose a time-period long enough to allow us to detect signal over noise but short enough to avoid an eventual change in slope. Because we have five separate realizations for each scenario, our signal to noise is sufficiently enhanced to permit use of a shorter period than that of [3]. We omit the first 10 years of simulated data to avoid inclusion of very short-term transients that occur immediately after a major climate shock, which would introduce spurious patterns in the inferred regional response.

For coefficient extraction from the CMIP3 $\mathrm{CO}_{2}$ doubling experiments, all of which were performed on fast-equilibrating slab ocean models, we use a shorter fitting period of 15 years for the regression. This follows the practice of [5], who used a period of 15 years when fitting output from a slab ocean model. For these experiments we estimate the equilibrium climate from the last 10 years of archived data. 


\section{Regional patterns for $\mathrm{CO}_{2}$ - and solar-forced runs}
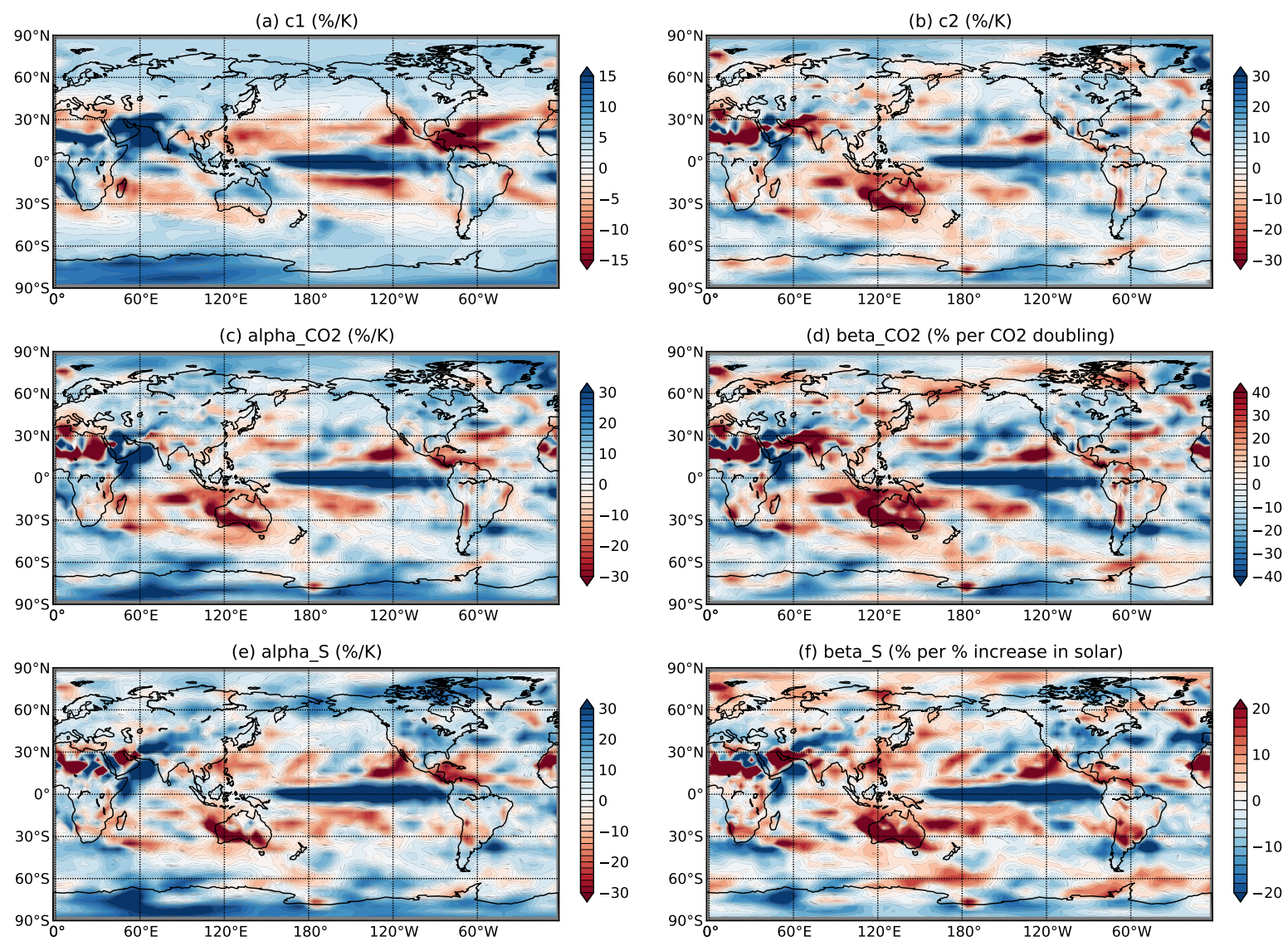

Supplementary Figure 9: Regional patterns of the coefficients (a) $c_{1}$, (b) $c_{2}$, (c) $\alpha$, (d) $\beta_{\mathrm{CO}_{2}}$, (e) $\alpha$ and (f) $\beta_{S}$. These coefficients are approximated using data from the instantaneous $\mathrm{CO}_{2}$ and solar experiments and the multi-millennial $\mathrm{CO}_{2}$ stabilization experiment. This figure is the same as Figure 7 in the manuscript, except that precipitation anomalies are expressed in $\%$ change rather than $\mathrm{cm} / \mathrm{yr}$.

In the manuscript, we show coefficients assuming precipitation anomalies are in units of $\mathrm{cm} / \mathrm{yr}$ (Figure 7 in the manuscript, which shows maps for the coefficients $c_{1}, c_{2}, \alpha_{\mathrm{CO}_{2}}, \beta_{\mathrm{CO}_{2}}$, $\alpha_{S}$ and $\beta_{S}$ ). Since global precipitation change is dominated by the tropics, the color schemes in those maps are dominated by this region. For an additional perspective, and to highlight relative changes in regional precipitation, we show the same maps with anomalies in units of percentage change in Supp. Fig. 9. These maps allow ready comparison to ClausiusClapeyron and emphasize the diversity of regional response. 


\section{Robustness across scenarios and models}

To determine whether our conclusions are robust, we repeat our analysis of coefficient correlations using output from a different forcing scenario and from different models. First, we analyze model output from a CCSM3 experiment we conducted where $\mathrm{CO}_{2}$ is increased instantaneously from $289 \mathrm{ppm}$ to $1400 \mathrm{ppm}$ (Supp. Fig. 10). Since we do not have the final equilibrium for this forcing scenario we can examine only $\alpha$ and $\beta_{\mathrm{CO}_{2}}$ and not $c_{1}$ and $c_{2}$. The patterns of $\alpha$ and $\beta_{\mathrm{CO}_{2}}$ are highly similar to those shown in the manuscript for an increase to $700 \mathrm{ppm}$ (Figure $7 \mathrm{c}$ and $\mathrm{d}$ of the manuscript), and the $\alpha$ and $\beta_{\mathrm{CO}_{2}}$ are again highly correlated $(\mathrm{r}=-0.91$; compare to Figure $6 \mathrm{~b}$ in the manuscript with $\mathrm{r}=-0.94$ for the 700 ppm run).

Second, we examine output from two models that have archived output from the CMIP3 "2xco2" experiment (UKMO_HADGEM1 and MRI_CGCM2.3.2a; see Supp. Figs. 11 and 12). (These two models roughly span the range of precipitation responses; however, our choice of models is somewhat arbitrary and ten more models which have archived data could be also be used.) The only archived output that is suitable for this analysis (i.e. that involves instantaneous-forcing change experiments) is derived from slab-ocean models with lower signal-to-noise. Because the fast-equilibrating slab-ocean models are run to equilibrium, however, we can also extract patterns and correlations for $c_{1}$ and $c_{2}$ for these studies. Slopes and intercepts are extracted using the first 15 years of model output, and equilibria are determined from the final 10 years. This experiment was run for 150 years for MRI_CGCM2.3.2a and 70 years for UKMO_HADGEM1, by which time a slab-ocean model is essentially fully equilibrated. The short equilibration time of the slab-ocean models precludes any removal of initial years to eliminate very short-timescale transients, so we would expect these responses to be somewhat more complicated. Despite these limitations, patterns and correlations of the coefficients $c_{1}, c_{2}, \alpha$ and $\beta_{\mathrm{CO}_{2}}$ support the findings of this manuscript. 

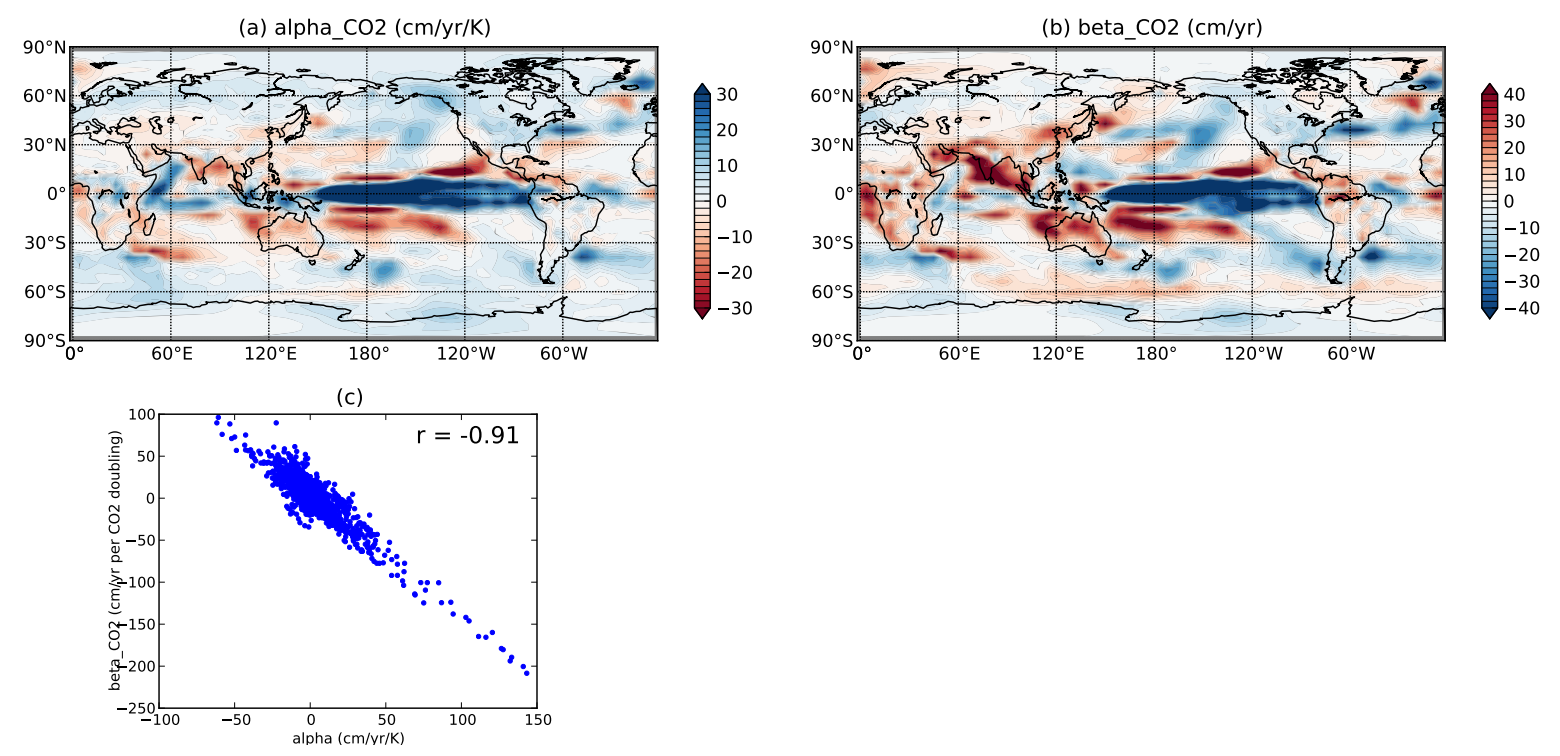

Supplementary Figure 10: Regional patterns of the coefficients (a) $\alpha$ and (b) $\beta_{\mathrm{CO}_{2}}$ estimated using output from a CCSM3 run where $\mathrm{CO}_{2}$ is increased instantaneously from 289 ppm to 1400 ppm, and the correlation between these coefficients (c). The maps are similar to those in manuscript Fig. 7 derived from the 700 ppm stabilization scenario discussed in the manuscript, and correlation of coefficients equally strong. (Compare to manuscript Figure $6 \mathrm{~b}$ ). Because we have no final equilibrium state for 1400 ppm, maps and correlations cannot be shown for $c_{1}$ and $c_{2}$ 

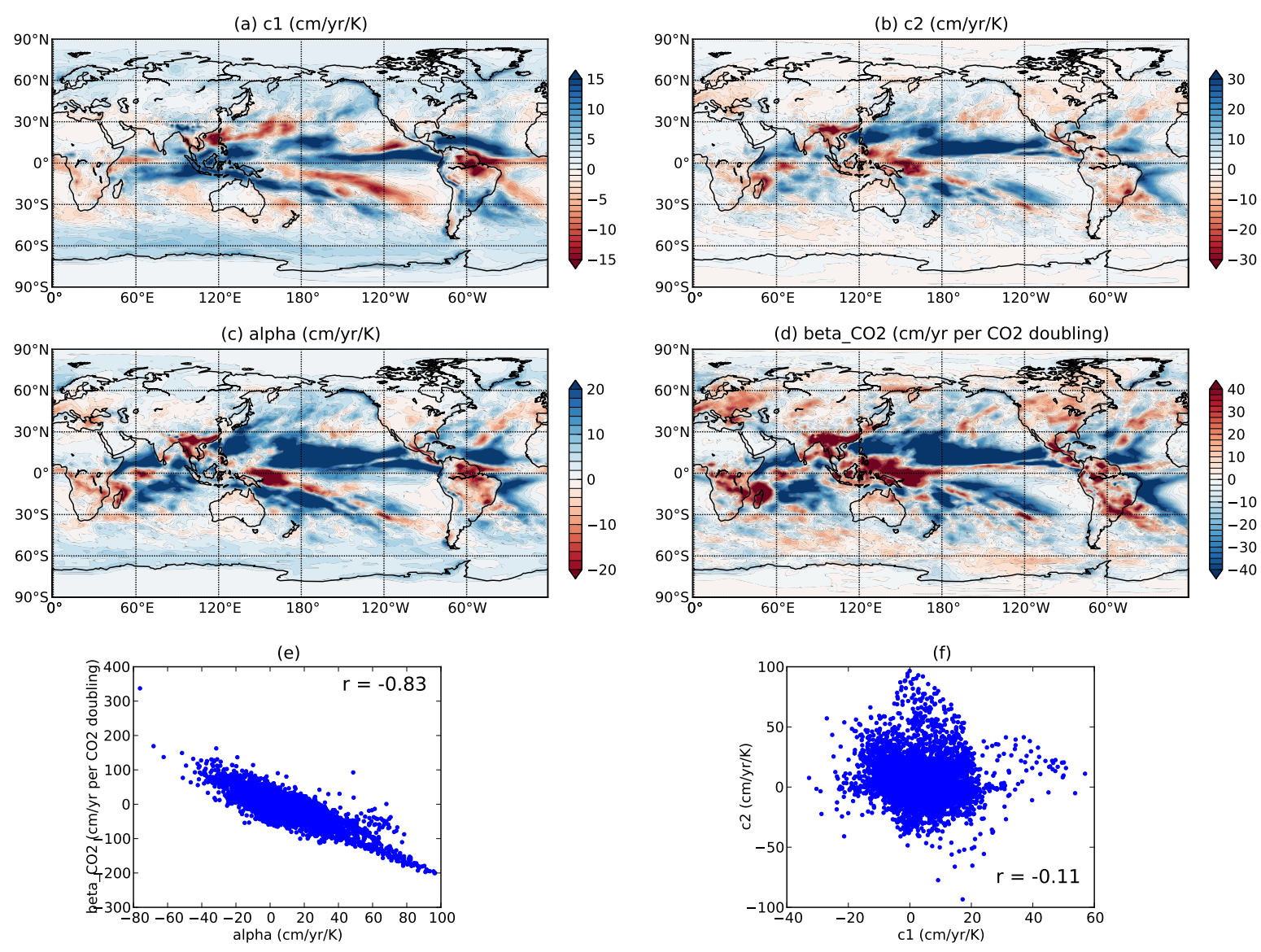

Supplementary Figure 11: Regional patterns of the coefficients (a) $c_{1}$ and (b) $c_{2}$, (c) $\alpha$ and (d) $\beta_{\mathrm{CO}_{2}}$ for the model UKMO_HADGEM1, based on data from the CMIP3 "2xco2" experiment. Panel (e) shows the correlation between coefficients $\alpha$ and $\beta_{\mathrm{CO}_{2}}$ and (f) the correlation between $c_{1}$ and $c_{2}$. The Pearson's correlation coefficient between $\alpha$ and $\beta_{\mathrm{CO}_{2}}(-0.83)$ is much larger than that between $c_{1}$ and $c_{2}(-0.11)$. 

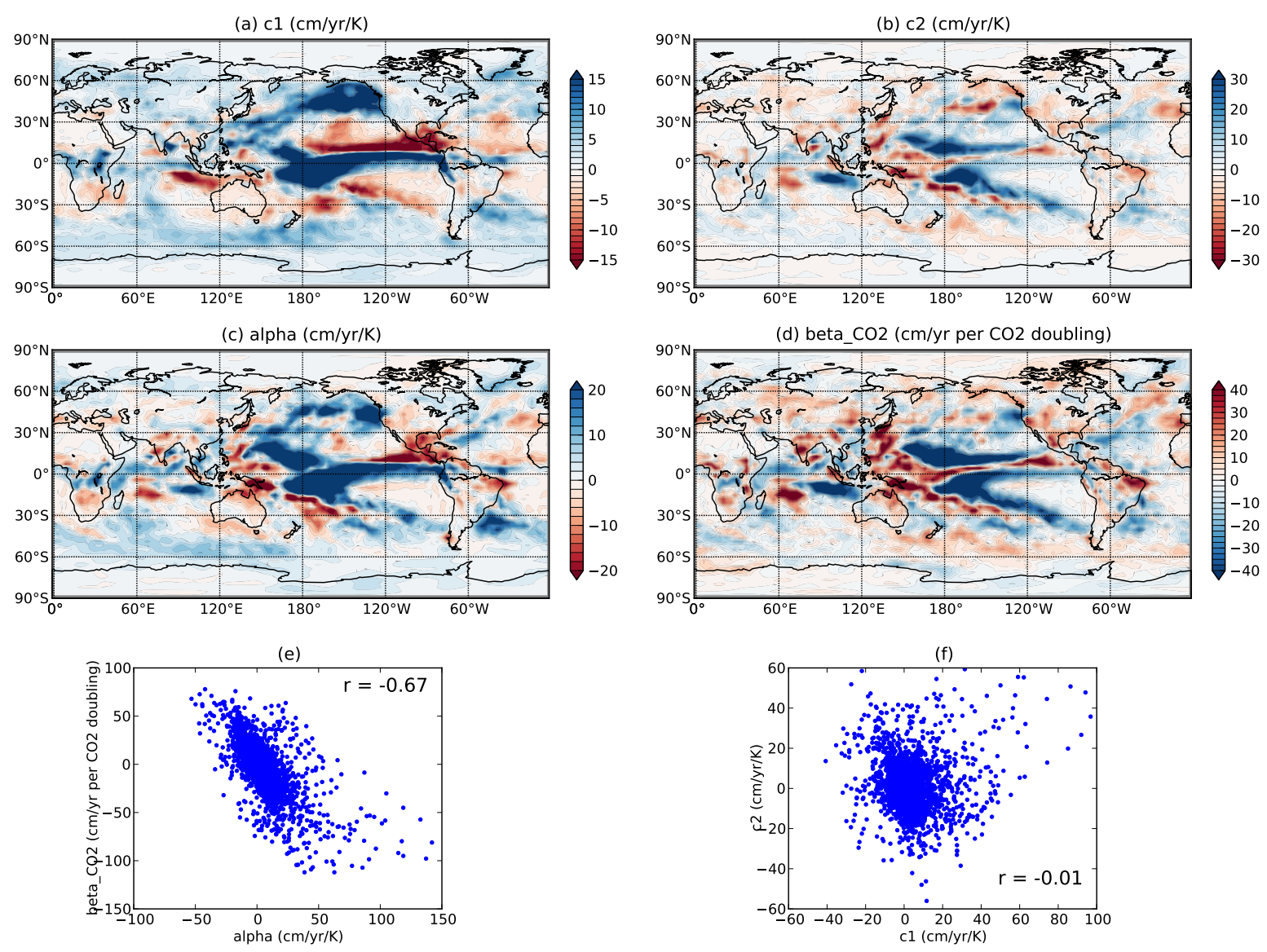

Supplementary Figure 12: Regional patterns of the coefficients (a) $c_{1}$ and (b) $c_{2}$, (c) $\alpha$ and (d) $\beta_{\mathrm{CO}_{2}}$ for the model MRI_CGCM2_3_2a, based on data from the CMIP3 "2xco2" experiment.

(e) shows the correlation between coefficients $\alpha$ and $\beta_{\mathrm{CO}_{2}}$, and (f) the correlation between $c_{1}$ and $c_{2}$. The Pearson's correlation coefficient between $\alpha$ and $\beta_{\mathrm{CO}_{2}}(-0.67)$ is much larger than that between $c_{1}$ and $c_{2}(-0.01)$. 


\section{Solar offset constancy over time}

In the manuscript we hypothesized that the offset between precipitation in solar- and $\mathrm{CO}_{2}$-forced climates climate of similar equilibrium temperature is due to a different physical mechanism than that which produces transient suppression in the $\mathrm{CO}_{2}$-forced case. The offset between solar and $\mathrm{CO}_{2}$ is almost universally positive and near-globally homogeneous. We show here that the offset is roughly constant over time, whereas the transient effects associated with ocean heat uptake vanish over time. Figure $8 \mathrm{c}$ in the manuscript shows the difference between precipitation for the solar forced run (with 2.55\% increase in solar forcing) and the $\mathrm{CO}_{2}$ forced run (which increases from $289 \mathrm{ppm}$ to $700 \mathrm{ppm}$ ), averaged over the first 300 years. To make the regional pattern of differences more clearly visible, we show it here with a different color scheme, and to show constancy, we show it for two different time periods. Supp. Fig. 13 (a) shows differences for the first 50 years, and (b) for the years 251-300 of the simulations. Over most of the globe, the solar-forced run shows $\approx 2-5$ $\mathrm{cm} / \mathrm{yr}$ more precipitation than does the $\mathrm{CO}_{2}$ forced case. The difference is stronger in the dry subtropics, where $\mathrm{CO}_{2}$-forced climates show actual precipitation decreases both in the transient period and at final equilibrium. This pattern appears broadly constant throughout the approach to equilibrium.
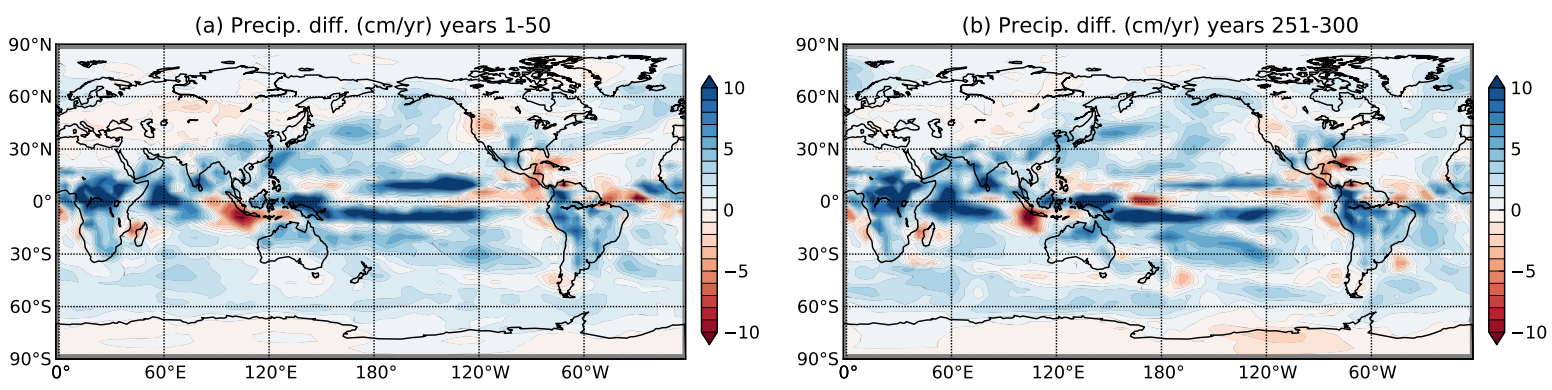

Supplementary Figure 13: The difference between precipitation anomalies in solar and $\mathrm{CO}_{2}$ runs, averaged over (a) the years 1-50 (when global mean temperature change is $\Delta T=2.1 \mathrm{~K}$ for both), and (b) 251-300 ( $\Delta T=2.7 \mathrm{~K}$ for both). 
[1] S.G. Yeager, C.A. Shields, W.G. Large, and J.J. Hack. The low-resolution CCSM3. Journal of Climate, 19:2545-2566, 2006.

[2] W.D. Collins, C.M. Bitz, M.L. Blackmon, G.B. Bonan, C.S. Bretherton, J.A. Carton, P. Chang, S.C. Doney, J.J. Hack, T.B. Henderson, J.T. Kiehl, W.G. Large, D.S. McKenna, B.D. Santer, and R.D. Smith. The Community Climate System Model: CCSM3. Journal of Climate, 19:2122-2143, 2006.

[3] T. Andrews and P.M. Forster. The transient response of global-mean precipitation to increasing carbon dioxide levels. Environmental Research Letters, 5(2), 2010.

[4] L. Cao, G. Bala, and K. Caldeira. Why is there a short-term increase in global precipitation in response to diminished $\mathrm{CO}_{2}$ forcing? Geophysical Research Letters, 38(L06703), 2011.

[5] G. Bala, K. Caldeira, and R. Nemani. Fast versus slow response in climate change: Implications for the global hydrological cycle. Climate Dynamics, 35:423-434, 2010. 